\title{
Regulating heart repair with cardiac-specific T lymphocytes
}

\author{
Ziad Mallat
}

Department of Medicine, Division of Cardiovascular Medicine, University of Cambridge, Cambridge, United Kingdom.

\begin{abstract}
Cardiac tissue necrosis secondary to coronary artery occlusion is one of the most common and deadly sterile injuries in developed countries. In this issue of the $J C I$, Rieckmann et al. identified and characterized antigenspecific CD4+ $T$ helper (Th) cells that developed in the context of myocardial infarction (MI) in mice. They showed that myosin heavy chain $\alpha$ (MYHCA) is a dominant cardiac autoantigen and that T cells with T cell receptor (TCR) specificity to MYHCA acquired a Treg phenotype when adoptively transferred into infarcted mice, which mediated a cardioprotective healing response. Thus, Rieckmann et al. showed that an acute ischemic insult to the heart, which induces sterile inflammation, promoted, rather than limited, protective T cell autoimmunity. Notably, strategies that support an antigenspecific Treg response may limit the immune-inflammatory response and promote cardiac repair after acute $\mathrm{MI}$.
\end{abstract}

example, under steady-state conditions in heart-draining mediastinal lymph nodes (medLNs), IRF8-dependent conventional DCs present cardiac MYHCA, which drives the expansion and differentiation of MYHCA-specific CD4 ${ }^{+} \mathrm{T}$ cells toward a protective Treg phenotype (4). Following acute MI, the release of proinflammatory cytokines (e.g., HMGB1, IL-1 $\alpha$, IL-1 $\beta$, IL-6), along with self-antigens, promotes DC maturation and activation and licenses MYHCA-specific T cells to adopt an (IL-17/IFN- $\gamma$ ) effector phenotype (4). Although this sequence may establish the background for increased susceptibility to post-MI autoimmune attack (5), in the absence of an autoimmune-prone background, tolerance generally remains intact, and pathologic cardiac autoimmunity fails to develop (6).

Rieckmann and colleagues studied the post-MI immune response in greater detail. They recovered MYHCA-specific $\mathrm{CD}^{+} \mathrm{T}$ (TCR-M) cells from MYHCATCR-transgenic mice and showed that after MI, the transferred cells improved heart function and promoted myocardial healing (7). The authors proposed that in the post-MI setting, the (preferential) differentiation and expansion of MYHCAspecific $\mathrm{CD}^{+} \mathrm{T}$ cells toward Tregs were responsible for maintaining immune tolerance and providing cardioprotective effects. This conclusion was based on an experiment in which the adoptively transferred TCR-M cells that proliferated and accumulated in the heart (but not in medLNs) displayed a higher percentage of Foxp $3^{+}$cells in mice with MI compared with sham-operated mice (7). In another mouse experiment, adoptively transferred conventional $\mathrm{T}$ helper (Tconv) cells converted to Tregs in situ within the heart and gave rise to most of the Foxp $3^{+}$TCR-M cardiac cells. However, the percentage (50\%) of TCR-M Tconv cells that acquired Foxp $3^{+}$ expression in the ischemic heart matched that of the sham-operated mice (7). Thus, the cardiac environment per se, whether 
ischemic or not, seems to favor the conversion of Tconv cells to Tregs. Given the cell scarcity, no further characterization of these heart-homing TCR-M cells was pursued. Instead, after transferring cells into sham-operated or infarcted mice, the authors performed gene expression profiling on medLN TCR-M cells. When cells from sham-operated mice were compared with those from infarcted mice, the transcription signature revealed enrichment of "prohealing" genes. However, no specific enrichment of Tregs was detected, and genes associated with T helper 17 (Th17) and $\mathrm{T}$ follicular helper cell responses were also enriched in MI medLN TCR-M cells compared with sham mice (7). Further characterization of TCR-M cells in the presence or absence of $\mathrm{MI}$ is warranted to determine the mechanisms responsible for their cardioprotective effects.

Rieckmann et al. also analyzed the endogenous $\mathrm{CD}^{+} \mathrm{T}$ cells that accumulated in the hearts and medLNs of mice after MI. The results revealed a unique repertoire signature for cardiac $\mathrm{T}$ cells with preferential expansion of clones that expressed a specific TCR $\beta$ chain variable region, TCRBV19 (7). It is interesting to note that similarly skewed $\mathrm{T}$ cell responses are induced after immunization of mice with cardiac myosin or heart extract (7) and have also been described in other settings associated with cardiovascular injury, including dilated cardiomyopathy in humans (8). The reasons behind this preferential usage of the TCR $\beta$ chain variable region remain unknown and merit further investigation. It will be interesting to examine whether endogenous $\mathrm{T}$ cell clones play beneficial or detrimental roles after MI. A recent study confirmed the occurrence of a skewed TCR repertoire in cardiac $\mathrm{T}$ cells of ischemic failing human hearts that was indicative of tissue-specific $\mathrm{T}$ cell expansion (9). Cardiac $\mathrm{T}$ cells from ischemic failing hearts displayed memory and effector phenotypes, with a predominance of Th1 and cytotoxic $\mathrm{CD} 8^{+}$ $\mathrm{T}$ cells (9), suggesting detrimental properties. Thus, although the immediate postMI setting may still be conducive to the development of a protective Treg response (7), a failure to maintain this T cell homeostasis during chronic ischemia may contribute to the progression to heart failure. Cells equivalent to IRF8-dependent DCs $\left(\right.$ CLEC9A $^{+}$DCs) and HLA-DR ${ }^{+}$macrophages showed close interactions with cardiac $\mathrm{T}$ cells in the failing hearts, indicating active antigen presentation. The use of advanced single-cell TCR-sequencing technologies and the development of screening methods with peptide-MHC may help identify associated (auto)antigens.

\section{Cardioprotective immunotherapies}

Can we harness post-MI $\mathrm{CD}^{+}{ }^{+} \mathrm{T}$ cell responses to improve patient stratification and develop cardioprotective immunotherapies? Rieckmann et al. combined CT with PET imaging using a radiolabeled C-X-C motif chemokine receptor 4 (CXCR4) ligand ( $\left[{ }^{68} \mathrm{Ga}\right]$ pentixafor) as a surrogate for $\mathrm{T}$ cell presence in the heart and medLNs. Interestingly, medLNs were significantly enlarged in patients with MI $(n=22)$ compared with medLNs of controls $(n=5)$. Despite the small size of the latter group and the fact that the "controls" were included in the study as part of an endocrinological investigation for suspected benign Conn's adenoma, the results are consistent with a local expansion of the $\mathrm{T}$ cell compartment in patients with MI. Additional studies are necessary to better characterize this response and examine whether it could provide any prognostic value (e.g., a better healing response and improved recovery of heart function at follow-up). A recent experimental study in mice and pigs showed that controlled CXCR4 blockade through bolus injections promoted Treg mobilization (from the spleen) and Treg homing to the heart and enhanced the immune-regulatory properties of these Tregs, leading to reduced infarct size and improved heart function (10). If and when CXCR4 blockade therapy is ready for use in patients with MI, it would be interesting to evaluate CXCR4 imaging to identify the most suitable candidates.

Treg-targeted therapies have the potential to both promote cardiac repair after MI (11-13), and limit the progression of atherosclerosis (14). An interesting Treg-promoting therapy that is currently being tested in patients with acute coronary syndromes involves the administration of low-dose IL-2 $(15,16)$, which is expected to selectively expand and activate Tregs at the expense of T effector cells (17). A big unknown, however, is whether Tregs can still be expand- ed and maintained locally within a hostile ischemic, necrotic, and inflammatory microenvironment. The study by Rieckmann et al. reassuringly suggests that such Treg-promoting therapies may effectively treat acute MI, given the apparent extraordinary ability of the cardiac environment to preserve, maintain, or even promote Treg differentiation, even in the presence of sterile injury and danger signals. Nevertheless, combination therapies that limit effector immune mechanisms $(16,18)$ may be necessary to ensure optimal results.

Finally, future studies should investigate why the cardiac environment is so unique in promoting Treg differentiation. In this regard, it is interesting to note that fibroadipogenic progenitors in skeletal muscle produce IL-33 to regulate muscle Treg homeostasis (19) and promote muscle repair (20). Cardiac fibroblasts are major sources of IL-33, whose production and release are further promoted by biomechanical stimuli and cell death (21). The hypothesis that IL-33 could, therefore, at least in part, favor local differentiation, activation, or maintenance of cardiac Tregs merits further exploration.

\section{Acknowledgments}

This work is supported by the British Heart Foundation (United Kingdom), INSERM (France), and the European Research Council.

Address correspondence to: Ziad Mallat, Department of Medicine, University of Cambridge, Addenbrookes Hospital, Hills Road, Cambridge CB2 OQQ, United Kingdom.Email:zm255@medschl.cam.ac.uk.

1. Lv H, et al. Impaired thymic tolerance to $\alpha$-myosin directs autoimmunity to the heart in mice and humans. J Clin Invest. 2011;121(4):1561-1573.

2. Prabhu SD, Frangogiannis NG. The biological basis for cardiac repair after myocardial infarction: from inflammation to fibrosis. Circ Res. 2016;119(1):91-112.

3. Legoux FP, et al. CD $4^{+} \mathrm{T}$ cell tolerance to tissuerestricted self antigens is mediated by antigenspecific regulatory $\mathrm{T}$ cells rather than deletion. Immunity. 2015;43(5):896-908.

4. Van der Borght K, et al. Myocardial infarction primes autoreactive $\mathrm{T}$ cells through activation of dendritic cells. Cell Rep. 2017;18(12):3005-3017.

5. Maisel A, Cesario D, Baird S, Rehman J, Haghighi P, Carter S. Experimental autoimmune myocarditis produced by adoptive transfer of splenocytes after myocardial infarction. Circ Res. 1998;82(4):458-463. 
6. Gottumukkala RV, et al. Myocardial infarction triggers chronic cardiac autoimmunity in type 1 diabetes. Sci Transl Med. 2012;4(138):138ra80.

7. Rieckmann M, et al. Myocardial infarction triggers cardioprotective antigen-specific T helper cell responses. J Clin Invest. 2019;129(11):4922-4936.

8. Blohm JH, et al. Detection of clonal T-cell-receptor (TCR) Vbeta rearrangements in explanted dilated cardiomyopathy hearts by semi-nested PCR, GeneScan, and direct sequencing. Med Sci Monit Basic Res. 2013;19:111-117.

9. Tang TT, et al. Pathologic T-cell response in ischaemic failing hearts elucidated by T-cell receptor sequencing and phenotypic characterization [published online ahead of print July 31, 2019]. Eur Heart J. https://doi.org/10.1093/eurheartj/ehz516.

10. Wang Y, et al. C-X-C motif chemokine receptor 4 blockade promotes tissue repair after myocardial infarction by enhancing regulatory $\mathrm{T}$ cell mobilization and immune-regulatory function. Circulation. 2019;139(15):1798-1812.
11. Tang TT, et al. Regulatory $\mathrm{T}$ cells ameliorate cardiac remodeling after myocardial infarction. Basic Res Cardiol. 2012;107(1):232.

12. Weirather J, et al. Foxp $3^{+} \mathrm{CD} 4^{+} \mathrm{T}$ cells improve healing after myocardial infarction by modulating monocyte/macrophage differentiation. Circ Res. 2014;115(1):55-67.

13. Saxena A, et al. Regulatory $\mathrm{T}$ cells are recruited in the infarcted mouse myocardium and may modulate fibroblast phenotype and function. Am J Physiol Heart Circ Physiol. 2014;307(8):H1233-H1242.

14. Ait-Oufella H, et al. Natural regulatory T cells control the development of atherosclerosis in mice. Nat Med. 2006;12(2):178-180.

15. Zhao TX, et al. Low-dose interleukin-2 in patients with stable ischaemic heart disease and acute coronary syndromes (LILACS): protocol and study rationale for a randomised, doubleblind, placebo-controlled, phase I/II clinical trial. BMJ Open. 2018;8(9):e022452.

16. Zhao TX, Mallat Z. Targeting the immune system in atherosclerosis: JACC state-of-the-art review. JAm Coll Cardiol. 2019;73(13):1691-1706.

17. Klatzmann D, Abbas AK. The promise of lowdose interleukin-2 therapy for autoimmune and inflammatory diseases. Nat Rev Immunol. 2015;15(5):283-294.

18. Zouggari $Y$, et al. B lymphocytes trigger monocyte mobilization and impair heart function after acute myocardial infarction. Nat Med. 2013;19(10):1273-1280.

19. Kuswanto W, et al. Poor repair of skeletal muscle in aging mice reflects a defect in local, interleukin-33-dependent accumulation of regulatory $\mathrm{T}$ cells. Immunity. 2016;44(2):355-367.

20. Burzyn D, et al. A special population of regulatory $\mathrm{T}$ cells potentiates muscle repair. Cell. 2013;155(6):1282-1295.

21. Sanada S, Hakuno D, Higgins LJ, Schreiter ER, McKenzie AN, Lee RT. IL-33 and ST2 comprise a critical biomechanically induced and cardioprotective signaling system. JClin Invest. 2007;117(6):1538-1549. 